Fetal Diagnosis and Therapy

\title{
False-Positive Rate in First-Trimester Screening Based on Ultrasound and Cell-Free DNA versus First-Trimester Combined Screening with Additional Ultrasound Markers
}

\author{
Karl Oliver Kagan ${ }^{\mathrm{a}}$ Vanessa Maier $^{\mathrm{a}}$ Jiri Sonek $^{\mathrm{b}, \mathrm{c}}$ Harald Abele $^{\mathrm{a}}$ \\ Kai Lüthgens ${ }^{d}$ Maximilian Schmide Philipp Wagner ${ }^{a}$ Markus Hoopmann ${ }^{a}$ \\ a Department of Women's Health, University Women's Hospital Tübingen, Tübingen, Germany; ${ }^{b}$ Fetal Medicine \\ Foundation USA, Dayton, $\mathrm{OH}, \mathrm{USA}^{\circ}{ }^{\mathrm{C}}$ Division of Maternal Fetal Medicine, Wright State University, Dayton, $\mathrm{OH}, \mathrm{USA}$; \\ ${ }^{d}$ Cenata GmbH, Tübingen, Germany; ${ }^{e}$ Roche Sequencing Solutions Inc., Ariosa Diagnostics Inc., San Jose, CA, USA
}

\section{Keywords}

Aneuploidy · First trimester $\cdot$ Nuchal translucency thickness $\cdot$ Nasal bone $\cdot$ Tricuspid flow $\cdot$ Ductus venosus flow $\cdot$ cfDNA · Trisomy 21

\begin{abstract}
Objective: To determine whether screening for trisomy 21 based on first-trimester combined screening (FTCS) with assessment of nasal bone (NB), tricuspid flow (TCF), and ductus venosus flow (DVF) results in similar false-positive rates compared to ultrasound and cell-free DNA (cfDNA) screening. Methods: This is a subanalysis of a prospective randomized controlled trial which was performed between October 2015 and December 2016. Pregnant women with a normal firsttrimester ultrasound examination at 11 to 13 weeks' gestation were randomized into two groups: (1) FTCS with assessment of the NB, TCF, and DVF (extended FTCS [eFTCS]), and (2) ultrasound + cfDNA screening. The false-positive rate in screening for trisomy 21 was defined as the primary outcome parameter. Results: The study population consisted of
\end{abstract}

\section{KARGER}

(c) 2018 S. Karger AG, Basel

E-Mail karger@karger.com

www.karger.com/fdt
688 women in each study arm. In the eFTCS group, the median delta fetal nuchal translucency thickness (NT) was $0.0 \mathrm{~mm}$, free beta-hCG and PAPP-A were 0.96 and $1.11 \mathrm{MoM}$, and NB, TCF, and DVF PIV were abnormal in $0.9,0.6$, and $7.0 \%$ cases. In the ultrasound + cfDNA group, the median delta NT was $0.0 \mathrm{~mm}$. In 10 pregnancies the cfDNA analysis was uninformative and the risk of trisomy 21 was based on eFTCS. There were no false-positive cases in the ultrasound + cfDNA group, whereas the false-positive rates were between 0.9 and $2.2 \%$ with eFTCS. Conclusion: Screening for trisomy 21 based on ultrasound + cfDNA has a lower false-positive rate than screening based on eFTCS.

(c) 2018 S. Karger AG, Basel

\section{Introduction}

Classical first-trimester combined screening (FTCS) based on maternal age, fetal nuchal translucency thickness (NT), and the serum markers free beta-hCG and PAPP-A has long been considered the standard of care in 
screening for trisomy 21,18 , and 13 [1, 2]. Test performance can be improved by the addition of other markers such as nasal bone (NB) assessment and Doppler evaluation of the tricuspid valve and ductus venosus flow (DVF) (extended FTCS [eFTCS]). These markers are abnormal in about $56-66 \%$ of the fetuses with trisomy 21 and in $0.6-3.2 \%$ of the euploid population, respectively [3-5]. In previous studies that combined FTCS with additional ultrasound markers, it was possible to increase the detection rate for trisomy 21 while reducing the false-positive rate [3-5]. In one study of about 1,800 euploid pregnancies and 93 fetuses with trisomy 21 using a risk threshold of $1: 50$, the detection rate increased from $86 \%$ based on the combination of maternal age and NT to $96 \%$ by the addition of additional ultrasound markers. At the same risk cutoff, the false-positive rate was halved [6].

The introduction of cell-free DNA (cfDNA) screening presents a challenge to classical FTCS. Using FTCS, the detection and false-positive rates for trisomy 21 are about $90-95 \%$ and $2.5-5 \%$, respectively. In contrast, these rates improve to 99 and $0.1 \%$ using cfDNA screening [7]. However, due to concerns about the potential cost of implementing universal cfDNA screening, many groups advocate a contingent approach. Here, the screening starts with classical FTCS as a primary test. Invasive testing is offered to those at the highest risk and cfDNA testing for those with a result in the intermediate range. Miltoft et al. [8] used a risk range of 1:100 to 1:1,000 to define the intermediate-risk group and reported detection and false-positive rates for trisomy 21 of 100 and $1.1 \%$, respectively.

We recently described a different approach, which combines a detailed ultrasound examination at 11-13 weeks' gestation with cfDNA screening in all but the highest-risk group [9]. Using this approach, invasive testing is offered if the NT is $>3.5 \mathrm{~mm}$ or if a fetal anomaly is found. In the remaining cases, the risk of common trisomies is computed based on cfDNA screening. In a randomized controlled trial which focused on the false-positive rate, we compared this model to classical FTCS. For the same risk threshold of 1:100, the false-positive rates were 0 and $2.5 \%$ for the ultrasound and cfDNA approach and FTCS, respectively. In this study, risk assessment in the FTCS group was done without the use of additional ultrasound markers.

In the present study, we set out to determine whether screening for trisomy 21 based on eFTCS can result in similar false-positive rates compared to the ultrasound and cfDNA approach.

\section{Methods}

This is a subanalysis of a prospective randomized controlled trial which was performed at a single site (Prenatal Medicine Department of the University of Tübingen, Germany) between October 2015 and December 2016. The population consisted of unselected patients referred for first-trimester screening. In the original study, we determined false-positive rates based on firsttrimester risk assessment for trisomy 21 using FTCS and compared them to an approach which combines ultrasound and cfDNA. Only cases with an NT $\leq 3.5 \mathrm{~mm}$ and cases without major defects were included in randomization to these two screening protocols. The results of the original study have been reported elsewhere [9].

In the current study, we compared the same two groups. However, this was done with inclusion of additional ultrasound markers which were examined in all cases. In the first group, the risk of aneuploidy was assessed using eFTCS: maternal and gestational age, fetal NT, maternal serum PAPP-A and free beta-hCG levels with the addition of NB, tricuspid flow (TCF), and DVF PIV. In the second group, the risk assessment was based on ultrasound findings and cfDNA screening. eFTCS screening was performed in those subjects where cfDNA was not informative. The cfDNA analysis was performed by Cenata $\mathrm{GmbH}$ (Tübingen, Germany) using the Harmony ${ }^{\circledR}$ Prenatal Test (Roche Inc., San Jose, CA, USA) as previously described [10-12].

Briefly, in our center, first-trimester risk assessment is performed at $11-13^{+6}$ weeks' gestation. It includes crown-rump length and NT measurements and a detailed ultrasound examination based on the ISUOG guidelines [13, 14]. In addition, we examine NB, TCF, and DVF PIV in all patients. All evaluations are performed according to the published guidelines $[4,5,15]$ by operators who are certified by the Fetal Medicine Foundation UK. The biochemical markers free beta-hCG and PAPP-A are also included into FTCS. These results are generally available on the following day. If the NT measurement is $>3.5 \mathrm{~mm}$ or if a fetal anomaly is identified, the patient is considered to be high risk and is offered invasive testing without a formal calculation of aneuploidy risk.

The cfDNA analysis performed was chromosome-selective using digital analysis of selected regions and included a simultaneous microarray-based assay of nonpolymorphic (chromosomes 13, 18, $21, \mathrm{X}$, and $\mathrm{Y}$ ) and polymorphic loci to estimate chromosome proportion and fetal fraction. The Fetal Fraction Optimized Risk of Trisomy Evaluation (FORTE) algorithm was used to provide patient-specific probability assessment for trisomy.

In cases where cfDNA analysis was performed, an additional tube of blood was collected in anticipation of the fact that in a certain proportion of the cases the cfDNA testing would be uninformative. This serum sample was centrifuged at $4,000 \mathrm{rpm}$ for $5 \mathrm{~min}$; the serum was aliquoted and stored at $-80^{\circ} \mathrm{C}$. In cases where no result was obtained by cfDNA analysis, the serum sample was gently thawed and free beta-hCG and PAPP-A levels were measured. The risk of trisomy 21 was then computed using eFTCS (reflex test) [16].

Irrespective of the method of risk calculation used (eFTCS or ultrasound + cfDNA testing), an invasive test was offered to the patient if the risk exceeded 1:100. This is in accordance with the guidelines of the UK National Screening Committee and the risk cutoff that the Harmony ${ }^{\circledR}$ Prenatal Test uses to define a "highprobability" result. All pregnant women and health care providers 
Table 1. Median risks of trisomy 21 based on different combinations of maternal age, NT, serum biochemistry, and the additional markers NB, TCF, and DVF

\begin{tabular}{llc}
\hline $\begin{array}{l}\text { Mode of risk calculation in the eFTCS and the } \\
\text { ultrasound + cfDNA group with failed results }\end{array}$ & eFTCS median risk (IQR) & $\begin{array}{l}\text { Ultrasound + cfDNA screening } \\
\text { median risk (IQR) }\end{array}$ \\
\hline MA + NT + SM & $1: 3,787(1: 1,605-1: 8,280)$ & $1: 10,000(1: 10,000-1: 10,000)$ \\
MA + NT + SM + NB & $1: 5,127(1: 2,461-1: 9,904)$ & $1: 10,000(1: 10,000-1: 10,000)$ \\
MA + NT + SM + TCF & $1: 5,267(1: 2,630-1: 9,947)$ & $1: 10,000(1: 10,000-1: 10,000)$ \\
MA + NT + SM + DVF & $1: 5,734(1: 2,630-1: 10,525)$ & $1: 10,000(1: 10,000-1: 10,000)$ \\
MA + NT + SM + NB + TCF & $1: 6,064(1: 2,943-1: 10,879)$ & $1: 10,000(1: 10,000-1: 10,000)$ \\
MA + NT + SM + NB + DVF & $1: 6,181(1: 2,817-1: 11,078)$ & $1: 10,000(1: 10,000-1: 10,000)$ \\
MA + NT + SM + TCF + DVF & $1: 6,157(1: 2,918-1: 11,074)$ & $1: 10,000(1: 10,000-1: 10,000)$ \\
MA + NT + SM + NB + TCF + DVF & $1: 6,481(1: 3,103-1: 11,425)$ & $1: 10,000(1: 10,000-1: 10,000)$ \\
MA + NT & $1: 1,287(1: 596-1: 2,270)$ & $1: 10,000(1: 10,000-1: 10,000)$ \\
MA + NT + NB & $1: 2,768(1: 1,252-1: 5,329)$ & $1: 10,000(1: 10,000-1: 10,000)$ \\
MA + NT + TCF & $1: 3,068(1: 1,438-1: 5,329)$ & $1: 10,000(1: 10,000-1: 10,000)$ \\
MA + NT + DVF & $1: 4,355(1: 1,875-1: 8,641)$ & $1: 10,000(1: 10,000-1: 10,000)$ \\
MA + NT + NB + TCF & $1: 5,267(1: 2,568-1: 9,951)$ & $1: 10,000(1: 10,000-1: 10,000)$ \\
MA + NT + NB + DVF & $1: 5,881(1: 2,558-1: 10,624)$ & $1: 10,000(1: 10,000-1: 10,000)$ \\
MA + NT + TCF + DVF & $1: 6,042(1: 2,715-1: 11,029)$ & $1: 10,000(1: 10,000-1: 10,000)$ \\
MA + NT + NB + TCF + DVF & $1: 6,219(1: 2,895-1: 11,409)$ & \\
\hline
\end{tabular}

cfDNA, cell-free DNA; DVF, ductus venosus flow; eFTCS, extended first-trimester combined screening; MA, maternal age; NB, nasal bone; NT, fetal nuchal translucency thickness; SM, serum markers; TCF, tricuspid flow.

were informed about the risk assessment as soon as the results were available.

Exclusion criteria were maternal age $<18$ years, crown-rump length measurement of $>84 \mathrm{~mm}$ or $<45 \mathrm{~mm}$, and multiple pregnancy, including vanishing twins.

The clinically relevant ultrasound and screening information was recorded in the Viewpoint database (GE Healthcare, Munich, Germany). Information on maternal weight and height was extracted from the patient records. Ethnicity, smoking status, and mode of conception was assessed using a questionnaire, and this information was also recorded in the Viewpoint database. Outcome data were added as soon as they became available.

Potential trial participants were given written information about the study and were counselled by a member of the perinatal medicine department staff. All women who agreed to participate in the trial gave written informed consent. Randomization was done by our local research institute in a 1:1 ratio, using computergenerated random numbers. The study was structured as an investigator-initiated trial. Approval for the study was obtained from the local ethics committee (No. 572/2015BO1). The original study was registered in the International Standard Randomised Controlled Trial Number registry (ISRCTN No. 11174071).

The primary-analysis population included only those participants for whom both the screening results and results of newborn examination or genetic testing (prenatal or postnatal) were available.

\section{Statistical Analysis}

The detailed power calculation of the original study is described elsewhere [9]. In summary, we assigned 700 women to each study arm in order to demonstrate a significant difference in the false-positive rate of both approaches.

Ultrasound plus cfDNA or Combined Screening with Additional Markers
In the eFTCS group and in those cases with an uninformative cfDNA result, we calculated the individual risk of trisomy 21 based on maternal age, fetal NT, serum markers (free beta-hCG and PAPP-A), and any combination of NB, TCF, and DVF PIV. The risk of trisomy 21 was computed based on the most recent Fetal Medicine Foundation UK algorithm. In the ultrasound + cfDNA group, the risk was calculated based on the FORTE algorithm.

Euploid cases with a risk of 1:100 or more were considered false-positive. Due to the very few false-positive cases that were observed in both study arms, a proper adjustment for the maternal age distribution in Germany was not performed. The false-positive rates in the FTCS groups with different combinations of additional markers were compared with those of the ultrasound + cfDNA group using Clopper and Pearson's 95\% confidence intervals.

\section{Results}

During the study period, 1,518 women were seen for first-trimester screening. In $31(2.0 \%)$ cases, we found an increased NT of $>3.5 \mathrm{~mm}$ and/or major fetal defects. In these cases, invasive testing rather than screening for trisomy 21 was performed. All pregnancies with trisomy 21 , 18 , and 13 fetuses were found within this group. Eightyseven $(5.9 \%)$ women declined study participation. The remaining 1,400 pregnancies were randomized into the 


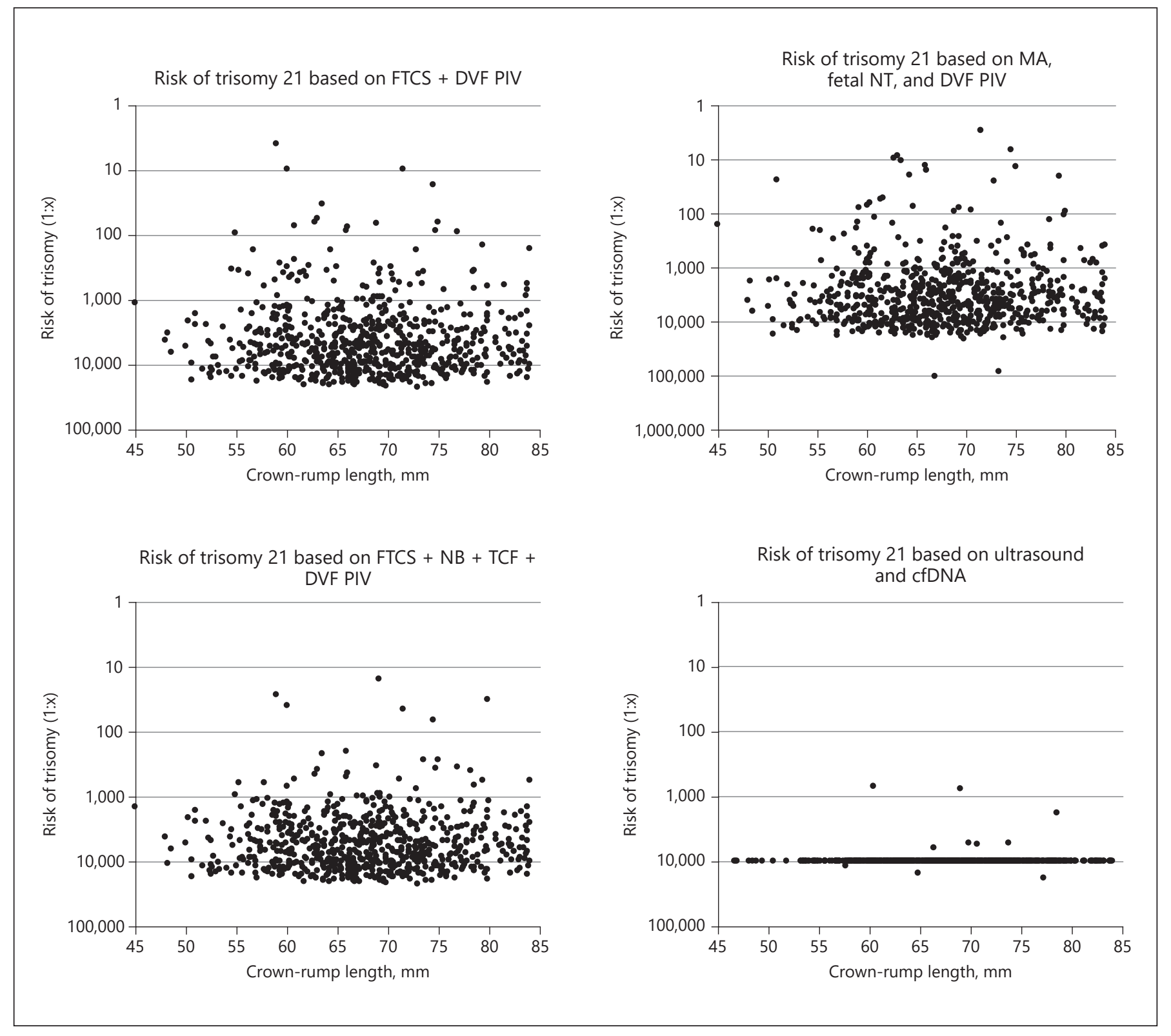

Fig. 1. Risks of trisomy 21 based on eFTCS and on the ultrasound + cfDNA approach. cfDNA, cell-free DNA; DVF, ductus venosus flow; eFTCS, extended first-trimester combined screening; FTCS, first-trimester combined screening; MA, maternal age; NB, nasal bone; NT, nuchal translucency thickness; TCF, tricuspid flow.

eFTCS $(n=699)$ and into the ultrasound + cfDNA $(n=$ $701)$ group. In 24 cases (1.7\%), it was not possible to obtain the outcome either due to loss to follow-up or due to a subsequent miscarriage or intrauterine fetal death. As a result, our final study group consisted of 688 women in each arm.

In the eFTCS group, median maternal age was 33.9 (IQR 30.7-36.7) years, gestational age was 12.7 (IQR
12.3-13.1) weeks, and maternal weight was 66.0 (IQR 59.1-74.3) kg. Median NT was 1.9 (IQR 1.6-2.1) mm, which corresponds to a median delta NT of 0.0 (IQR -0.2 to 0.2$) \mathrm{mm}$. Median free beta-hCG and PAPP-A levels were 0.96 (IQR 0.64-1.44) and 1.11 (IQR 0.76-1.51) MoM, respectively. NB and TCF were classified as abnormal in $6(0.9 \%)$ and $4(0.6 \%)$ cases, respectively. DVF was reversed in 18 fetuses (2.6\%). Median PIV was 1,037, and 
Table 2. Proportion of euploid cases with a high and an intermediate risk in screening by eFTCS and by a combination of ultrasound and cfDNA

\begin{tabular}{|c|c|c|c|c|}
\hline $\begin{array}{l}\text { Mode of risk calculation in the } \\
\text { eFTCS and the ultrasound }+ \text { cfDNA } \\
\text { group with failed results } \\
\text { Risk of trisomy } 21\end{array}$ & \multicolumn{2}{|c|}{ eFTCS, $n(\%)(95 \%$ CI) } & \multicolumn{2}{|c|}{ Ultrasound + cfDNA, $n(\%)(95 \% \mathrm{CI})$} \\
\hline $\mathrm{MA}+\mathrm{NT}+\mathrm{SM}$ & $17(2.5)(1.4-3.9)$ & $79(11.5)(9.2-14.1)$ & $0(0)(0.0-0.5)$ & $2(0.3)(0-1.0)$ \\
\hline $\mathrm{MA}+\mathrm{NT}+\mathrm{SM}+\mathrm{NB}$ & $6(0.9)(0.3-1.9)$ & $58(8.4)(6.5-10.8)$ & $0(0)(0.0-0.5)$ & $0(0)(0.0-0.5)$ \\
\hline $\mathrm{MA}+\mathrm{NT}+\mathrm{SM}+\mathrm{NB}+\mathrm{TCF}$ & $6(0.9)(0.3-1.9)$ & $38(5.5)(3.9-7.5)$ & $0(0)(0.0-0.5)$ & $0(0)(0.0-0.5)$ \\
\hline $\mathrm{MA}+\mathrm{NT}+\mathrm{SM}+\mathrm{NB}+\mathrm{DVF}$ & $6(0.9)(0.3-1.9)$ & $42(6.1)(4.4-8.2)$ & $0(0)(0.0-0.5)$ & $0(0)(0.0-0.5)$ \\
\hline $\mathrm{MA}+\mathrm{NT}+\mathrm{SM}+\mathrm{TCF}+\mathrm{DVF}$ & $8(1.2)(0.5-2.3)$ & $43(6.3)(4.6-8.3)$ & $0(0)(0.0-0.5)$ & $0(0)(0.0-0.5)$ \\
\hline $\mathrm{MA}+\mathrm{NT}+\mathrm{SM}+\mathrm{NB}+\mathrm{TCF}+\mathrm{DVF}$ & $6(0.9)(0.3-1.9)$ & $25(3.6)(2.4-5.3)$ & $0(0)(0.0-0.5)$ & $0(0)(0.0-0.5)$ \\
\hline $\mathrm{MA}+\mathrm{NT}$ & $17(2.5)(1.4-3.9)$ & $267(38.8)(35.1-42.6)$ & $0(0)(0.0-0.5)$ & $4(0.6)(0.2-1.5)$ \\
\hline $\mathrm{MA}+\mathrm{NT}+\mathrm{NB}$ & $10(1.5)(0.7-2.7)$ & $126(18.3)(15.5-21.4)$ & $0(0)(0.0-0.5)$ & $2(0.3)(0-1.0)$ \\
\hline $\mathrm{MA}+\mathrm{NT}+\mathrm{TCF}+\mathrm{DVF}$ & $15(2.2)(1.2-3.6)$ & $38(5.5)(3.9-7.5)$ & $0(0)(0.0-0.5)$ & $0(0)(0.0-0.5)$ \\
\hline $\mathrm{MA}+\mathrm{NT}+\mathrm{NB}+\mathrm{TCF}+\mathrm{DVF}$ & $12(1.7)(0.9-3.0)$ & $28(4.1)(2.7-5.8)$ & $0(0)(0.0-0.5)$ & $0(0)(0.0-0.5)$ \\
\hline
\end{tabular}

In cases with an uninformative cfDNA test result, the risk of trisomy 21 is based on the respective FTCS approach. cfDNA, cell-free DNA; DVF, ductus venosus flow; eFTCS, extended first-trimester combined screening; FTCS, first-trimester combined screening; MA, maternal age; NB, nasal bone; NT, fetal nuchal translucency thickness; SM, serum markers; TCF, tricuspid flow.

in $48(7.0 \%)$ cases the DVF PIV was above the 95th centile.

In the ultrasound + cfDNA group, median maternal and gestational age were 33.9 (IQR 31.0-36.8) years and 12.7 (IQR 12.4-13.1) weeks, respectively. Median maternal weight was 65.4 (IQR 59.0-73.7). In this group, fetal NT and median delta NT were 1.8 (IQR 1.6-2.1) and 0.0 (IQR -0.2 to 0.2$) \mathrm{mm}$, respectively. In $10(1.4 \%)$ pregnancies, the cfDNA analysis was uninformative and the risk calculation for trisomy 21 was based on eFTCS. Fetal $\mathrm{NT}$, free beta-hCG, and PAPP-A were 1.7 (IQR 1.4-2.1) $\mathrm{mm}, 0.66$ (IQR 0.37-0.90) MoM, and 0.73 (IQR 0.671.23) MoM, respectively. None of these cases had an abnormal NB, TCF, or DVF. Median DVF PIV was 1,020, and in all cases the measurements were below the 95th centile.

The risks of trisomy 21 are summarized in Table 1 . The median risk of trisomy 21 with classical FTCS was 1:3,787. If the risk was computed based on eFTCS with one additional ultrasound marker, the median risk ranged between $1: 5,127$ and 1:5,734 and differed slightly according to the marker used. Depending on the combination of markers used, the median risks with two additional markers ranged from $1: 6,064$ to $1: 6,181$. When all three additional markers were used, the median risk was 1:6,418. If the risks of trisomy 21 were calculated without maternal serum biochemical markers, they ranged between 1:2,787 and 1:6,219 depending on the combination of markers used.

In the ultrasound + cfDNA group, median risk was $1: 10,000$ irrespective of the mode of risk calculation in those with uninformative cfDNA test results. Figure 1 demonstrates three examples of risk distributions in screening for trisomy 21, including eFTCS, and the ultrasound + cfDNA approach.

Table 2 shows the false-positive rates defined as risk of trisomy 21 above 1:100 and the proportion of cases with a risk between 1:100 and 1:1,000 according to the mode of risk calculation. While there were no false-positive cases in the ultrasound + cfDNA group, with eFTCS, the false-positive rates were between 0.9 and $3.2 \%$. If the risk was computed based on fetal NT and one, two, or three additional markers but without the serum markers, the false-positive rate was significantly higher than with the ultrasound + cfDNA approach. Furthermore, in the ultrasound + cfDNA group, only $0-0.6 \%$ of cases had a risk between 1:100 and 1:999. In these cases, the cfDNA anal- 
ysis was uninformative and the risk of trisomy 21 was computed based on eFTCS. In contrast, this proportion ranged between 3.6 and $38.8 \%$ if the risk assessment was based on various permutations of FTCS.

\section{Discussion}

\section{Main Findings of Our Study}

In this study, we compared screening for trisomy 21 based on eFTCS with a model that is based on ultrasound + cfDNA screening. The false-positive rate was lowest with the latter approach. This was true after inclusion of one, two, or even three additional ultrasound markers. However, these differences were not statistically significant. If maternal serum biochemical markers were excluded from eFTCS screening, the false-positive rates became statistically significantly higher than those associated with ultrasound + cfDNA screening. Furthermore, the proportion of cases with a risk between 1:100 and 1:999 was significantly larger with any of the eFTCS models compared with the ultrasound + cfDNA screening approach.

We focused on the false-positive rate as the primary outcome parameter because in terms of absolute numbers, this is the most crucial screening parameter that is of major importance to physicians, health authorities, and patients alike. Of note, in our study all fetuses with trisomy 21,18 , and 13 were identified by ultrasound.

\section{Comparison with Previous Studies}

To our knowledge, this is the first publication which directly compares the false-positive rates of eFTCS to an ultrasound + cfDNA approach. However, several reports exist that evaluate the screening performance of eFTCS. Three publications that were based on approximately 20,000 normal pregnancies and 122 pregnancies affected by trisomy 21 evaluated the change in screening efficiency by adding assessment of NB, TCF, and DVF in a dichotomous way. Specifically, in each study, one of the additional ultrasound markers was used on a contingent basis if the FTCS risk was between 1:50 to 1:1,000 [3-5]. The false-positive rate was reduced to about $2.5 \%$, which is half that of classical FTCS. At the same time, the detection rate for trisomy 21 increased to about $95 \%$. Maiz et al. [15] used DVF PIV in addition to FTCS. The authors reported a detection rate of $94 \%$ for trisomy 21 and a false-positive rate of $1.6 \%$. We assessed the change in test performance in screening for trisomy 21 based on maternal age, maternal serum biochemical markers, and fetal NT if one, two, or three ultrasound markers (NT, TCF, DVF) were added. For a risk cutoff of 1:50, the detection rate for trisomy 21 increased from $86 \%$ with one of the markers to 90 and $96 \%$ with two and three markers, respectively [6]. For the same cutoff, the detection rate for trisomy 18, 13, triploidy, and Turner syndrome was 96\% [17]. Other study groups have found similar results [18-20].

\section{Limitations of Our Study}

We acknowledge that our study has some limitations. The original study was powered to demonstrate a significant difference between FTCS and ultrasound + cfDNA screening. In this subanalysis, eFTCS was used, which has a lower false-positive rate $(0.9-2.2 \%)$. Thus, we were not able to demonstrate significant differences between the study groups. Furthermore, the ultrasound examination was carried out by prenatal medicine experts. It could be argued that in a more general setting, such ultrasound results cannot be achieved. However, the ultrasound examination was carried out according to the ISUOG guidelines and thus should be generally reproducible [14].

In addition, in both study arms, we only focused on the risk of trisomy 21 . Inclusion of the risk of trisomy 18 and 13 may result in a higher false-positive rate. However, we believe that affected fetuses are identified by the detailed ultrasound examination and excluded from the screening process due to the presence of major defects.

\section{Implications for Clinical Practice}

In a recent meta-analysis, Gil et al. [7] summarized the test performance of cfDNA screening for trisomy 21. The updated detection and false-positive rates were 99.7 and $0.04 \%$, respectively. However, trisomy 21 only covers about half of the chromosomal abnormalities [21]. If the standard also includes chromosomal microarray analysis, this proportion is even less [22]. Therefore, a first-trimester ultrasound examination, which includes a detailed fetal anatomic evaluation and NT measurement, should always be performed. If an anomaly is present or if fetal NT is increased, invasive testing including chromosomal microarray analysis should be offered [14]. The specific NT threshold that should be used for this purpose is still under discussion. Maya et al. [23] found that $2.2 \%$ of cases with an NT $\geq 3.5 \mathrm{~mm}$ had a chromosomal defect that can only be detected by microarray analysis. In the cases with an NT measurement between 3.0 and $3.4 \mathrm{~mm}$ or $\leq 2.9 \mathrm{~mm}$, the proportion was 1.8 and $0.9 \%$, respectively. These data suggest that a threshold of $3.0 \mathrm{~mm}$ may be more appropriate. However, 
this approach would lead to an increase in the rate of invasive testing.

For all cases other than those with fetal defects and/ or an increased NT, the results of the original randomized controlled trial and of this subanalysis indicate that cfDNA screening is preferable to eFTCS or classical FTCS due to the lower false-positive rate in screening for trisomy 21 . Still, assessment of the additional ultrasound markers is useful in screening for other fetal abnormalities such as cardiac defects [19]. In circumstances where essentially universal cfDNA screening cannot be implemented, such as due to financial constraints, screening based on measurement of maternal serum markers (free beta-hCG and PAPP-A) and NT as well as assessment of $\mathrm{NB}, \mathrm{TCF}$, and DVF PIV is useful to reduce the false-positive rate. In contrast to our previous study, in this study we did not detect differences in the false-positive rate whether one, two, or three additional markers were used [6]. This is most probably due to the small numbers.

\section{Conclusion}

This study shows that screening for trisomy 21 based on a detailed ultrasound examination followed by cfDNA screening results in the lowest false-positive rate that is currently possible. Even by adding the results of NT, TCF, and DVF examination to FTCS, the false-positive rate remains higher.

\section{Acknowledgments}

Cenata GmbH (Tübingen, Germany) performed the cfDNA analysis. Roche/Ariosa Diagnostics Inc. (San Jose, CA, USA) provided the kits for the Harmony ${ }^{\circledR}$ Prenatal Test.

\section{Disclosure Statement}

M. Schmid is an employee of Roche Sequencing Solutions Inc.

\section{References}

1 Kagan KO, Sonek J, Wagner P, Hoopmann M: Principles of first trimester screening in the age of non-invasive prenatal diagnosis: screening for other major defects and pregnancy complications. Arch Gynecol Obstet 2017;296:635-643.

2 Santorum M, Wright D, Syngelaki A, Karagioti N, Nicolaides KH: Accuracy of first-trimester combined test in screening for trisomies 21, 18 and 13. Ultrasound Obstet Gynecol 2017;49:714-720.

3 Maiz N, Valencia C, Kagan KO, Wright D, Nicolaides KH: Ductus venosus Doppler in screening for trisomies 21,18 and 13 and Turner syndrome at 11-13 weeks of gestation. Ultrasound Obstet Gynecol 2009;33:512-517.

4 Kagan KO, Cicero S, Staboulidou I, Wright D, Nicolaides KH: Fetal nasal bone in screening for trisomies 21,18 and 13 and Turner syndrome at 11-13 weeks of gestation. Ultrasound Obstet Gynecol 2009;33:259-264.

5 Kagan KO, Valencia C, Livanos P, Wright D, Nicolaides KH: Tricuspid regurgitation in screening for trisomies 21,18 and 13 and Turner syndrome at $11+0$ to $13+6$ weeks of gestation. Ultrasound Obstet Gynecol 2009; 33:18-22.

6 Abele H, Wagner P, Sonek J, Hoopmann M, Brucker S, Artunc-Ulkumen B, et al: First trimester ultrasound screening for Down syndrome based on maternal age, fetal nuchal translucency and different combinations of the additional markers nasal bone, tricuspid and ductus venosus flow. Prenat Diagn 2015; 35:1182-1186.
7 Gil MM, Accurti V, Santacruz B, Plana MN, Nicolaides KH: Analysis of cell-free DNA in maternal blood in screening for aneuploidies: updated meta-analysis. Ultrasound Obstet Gynecol 2017;50:302-314.

8 Miltoft CB, Rode L, Ekelund CK, Sundberg $\mathrm{K}$, Kjaergaard S, Zingenberg $\mathrm{H}$, et al: Contingent first-trimester screening for aneuploidies with cell-free DNA in a Danish clinical setting. Ultrasound Obstet Gynecol 2018;51: 470-479.

9 Kagan KO, Sroka F, Sonek J, Abele H, Lüthgens K, Schmid M, et al: First-trimester risk assessment based on ultrasound and cell-free DNA vs combined screening: a randomized controlled trial. Ultrasound Obstet Gynecol 2018:51:437-444.

10 Juneau K, Bogard PE, Huang S, Mohseni M, Wang ET, Ryvkin P, et al: Microarray-based cell-free DNA analysis improves noninvasive prenatal testing. Fetal Diagn Ther 2014;36: 282-286.

11 Sparks AB, Wang ET, Struble CA, Barrett W, Stokowski R, McBride C, et al: Selective analysis of cell-free DNA in maternal blood for evaluation of fetal trisomy. Prenat Diagn 2012;32:3-9.

12 Sparks AB, Struble CA, Wang ET, Song K, Oliphant A: Noninvasive prenatal detection and selective analysis of cell-free DNA obtained from maternal blood: evaluation for trisomy 21 and trisomy 18. Am J Obstet Gynecol 2012;206:319.e1-e9.
13 Wright D, Kagan KO, Molina FS, Gazzoni A, Nicolaides KH: A mixture model of nuchal translucency thickness in screening for chromosomal defects. Ultrasound Obstet Gynecol 2008;31:376-383.

14 Salomon LJ, Alfirevic Z, Bilardo CM, Chalouhi GE, Ghi T, Kagan KO, et al: ISUOG practice guidelines: performance of first-trimester fetal ultrasound scan. Ultrasound $\mathrm{Ob}$ stet Gynecol 2013;41:102-113.

15 Maiz N, Wright D, Ferreira AFA, Syngelaki A, Nicolaides KH: A mixture model of ductus venosus pulsatility index in screening for aneuploidies at 11-13 weeks' gestation. Fetal Diagn Ther 2012;31:221-229.

16 Grati FR, Kagan KO: Rate of no result in cellfree DNA testing and its influence on test performance metrics. Ultrasound Obstet Gynecol 2017;50:134-137.

17 Wagner P, Sonek J, Hoopmann M, Abele H, Kagan KO: First-trimester screening for trisomies 18 and 13, triploidy and Turner syndrome by detailed early anomaly scan. Ultrasound Obstet Gynecol 2016;48:446-451.

18 Ghaffari SR, Tahmasebpour AR, Jamal A, Hantoushzadeh S, Eslamian L, Marsoosi V, et al: First-trimester screening for chromosomal abnormalities by integrated application of nuchal translucency, nasal bone, tricuspid regurgitation and ductus venosus flow combined with maternal serum free $\beta$-hCG and PAPP-A: a 5-year prospective study. Ultrasound Obstet Gynecol 2012;39:528-534.
Ultrasound plus cfDNA or Combined Screening with Additional Markers 
19 Karadzov-Orlic N, Egic A, Milovanovic Z, Marinkovic M, Damnjanovic-Pazin B, Lukic $\mathrm{R}$, et al: Improved diagnostic accuracy by using secondary ultrasound markers in the firsttrimester screening for trisomies 21,18 and 13 and Turner syndrome. Prenat Diagn 2012;32: 638-643.

20 Hsiao CH, Cheng PJ, Shaw SWS, Hsu JJ, Chen RC, Tseng YJ, et al: Extended first-trimester screening using multiple sonographic mark- ers and maternal serum biochemistry: a fiveyear prospective study. Fetal Diagn Ther 2014;35:296-301.

21 Grati FR, Barlocco A, Grimi B, Milani S, Frascoli G, Di Meco AM, et al: Chromosome abnormalities investigated by non-invasive prenatal testing account for approximately 50\% of fetal unbalances associated with relevant clinical phenotypes. Am J Med Genet 2010; 152A:1434-1442.
22 Shani H, Goldwaser T, Keating J, Klugman S: Chromosomal abnormalities not currently detected by cell-free fetal DNA: a retrospective analysis at a single center. Am J Obstet Gynecol 2016;214:729.e1-e11.

23 Maya I, Yacobson S, Kahana S, Yeshaya J, Tenne T, Agmon-Fishman I, et al: Cut-off value of nuchal translucency as indication for chromosomal microarray analysis. Ultrasound Obstet Gynecol 2017;50:332-335. 